\title{
BIT ERROR ANALYSIS OF NEW GENERATION WIRELESS TRANSCEIVERS
}

\author{
Abhishek Mitra \\ Indian Institute of Information Technology, Allahabad 211002, India, amitra@iiita.ac.in
}

\begin{abstract}
There has been a spurt of activity in the development of digital wireless transceivers especially those operating in the ISM (Industry Scientific Medical) bands. This is attributed to the fact that wireless systems have become more reliable, compact and easy to develop than ever before, leading to a well-perceived ubiquity. Most manufacturers do not document the error related performance and characteristics of their devices, thus entailing a detailed analysis by the developer or the user. In this paper we highlight tests to measure the bit error related performance of these new generation transceivers, with special emphasis on the TRF 6900A [3] transceiver from Texas Instruments, a popular binary FSK (Frequency Shift Keying) device. The test bed consists of a wireless modem based on the transceiver, and a personal computer executing a ' $\mathrm{C}$ ' language code for baseband control and data source - sink, in order to test the device in various configurations and conditions. Our test analyses bit error rate and tries to highlight its relationship to various parameters such as attenuation, interference, occlusions, and modulation index, which in turn helps to evaluate the performance of a wireless device. This method can be easily extended to evaluate various other similar wireless transceivers offered in the market.
\end{abstract}

Keywords - Wireless transceiver, bit error test, wireless modem, FSK.

\section{INTRODUCTION}

There has been a long felt need to untether the computer and its Input / Output devices. Various emerging standards like Bluetooth, Home RF, IEEE 802.11, etc have been proposed to address this need. Numerous manufactures, including fabless ones have come around with their arsenal of RF transceivers which operate at various frequencies such as $450 \mathrm{MHz}, 920$ $\mathrm{MHz}, 2.4 \mathrm{GHz}$, all part of the designated ISM (Industrial Scientific Medical) band. Due to operation of various devices in these unregulated bands, the transceivers are prone to interference. Spread Spectrum and Error Correction techniques are a prerequisite to operate devices in ISM bands to minimize any errors in transmission and to avoid channel contention for too long. Some common applications of ISM band devices include wireless peripherals like keyboard and mouse, Bluetooth enabled devices, wireless data acquisition devices especially electricity utility meters, security devices such as camera, car security systems, alarms, cordless phones, etc and the list is ever growing. RF transceivers are also used for wireless computer communications, mobile Internet connections such as in 'Ricochet', and in networks such as Bluetooth and IEEE 802.11. This paper concentrates on the transceiver ICs (Integrated Circuits) used for similar purposes, employing modulation schemes such as FSK, ASK (Amplitude Shift Keying), GMSK (Gaussian Minimum Shift Keying), [1],[5] etc. The transmit and receive data lines of a transceiver can be connected to a computer through a serial communications port, usually the ubiquitous 'RS $232 \mathrm{C}$ '. A ' $C$ ' language program transmits a pre-defined file through the wireless device to another computer. The transmitted file is compared offline to identify errors in the received data. In case of real-time analysis, small pre defined packets are transmitted continuously, which are tested at the receiver end for errors. This technique of sending packets is useful to calculate error rates in a mobile scenario, and to perform analysis 'on the fly'. The TRF6900 transceiver needs training at fixed time intervals, wherein data transmission is interrupted for a while. Our program takes care of this fact and trains the receiver at regular intervals. During the training session a 'store and hold' capacitor in the modem is charged to the average demodulation voltage level that persists when a series of alternating zeros and ones are received. Subsequent to the training, NRZ (Non Return to Zero) encoded data may be received without any worries of baseline wander, and a long series of zeros or ones can be correctly identified. Signal levels of the RS $232 \mathrm{C}$ port and TRF 6900A are incompatible therefore a level converter converts the voltage levels from RS 232C (+-9V) to CMOS $(3.3 \mathrm{~V})$ levels. However the reverse may not be necessary as the serial port can readily distinguish data received at CMOS $3.3 \mathrm{~V}$ levels. Our method eliminates need for additional equipments like BERTs (Bit Error Rates Testers), and has been used to test the transceiver for errors due to various parameters.

\section{THE WIRELESS TRANSCEIVER}

A typical wireless transceiver consists of transmitter and receiver subsections embedded on a single chip. A transmitter 
generally comprises of a VCO (Voltage Controlled Oscillator), PLL/DDS (Phase Locked Loop /Direct Digital Synthesizer) [3], power amplifier, and the modulation logic, while the receiver incorporates the LNA (Low Noise Amplifier), mixer, demodulator, and a comparator (slicer). Within most transceiver architectures, the VCO and PLL are shared between transmit and receive modes; consequently these devices operate in half duplex. The crystal oscillator serves as a reference for the PLL or the DDS, as the case may be. After power up, the transceiver needs to be programmed to configure the operating frequency, data rate, output power, transmit or receive mode, and various other parameters specific to the type of the device. Most modems use antennas embedded on the circuit board itself, leading to a compact design that can be nicely enclosed in a flat and compact structure a PC-Card for example. The Texas Instrument's TRF6900A boasts many useful features which include ability to handle both NRZ (Non Return to Zero) and constant DC (Manchester) encoded data, fast TX/RX (Transmit / Receive) switching, and a DDS based binary FSK modulator. This device employs a quadrature demodulator for FSK demodulation. An RSSI (Radio Strength Signal Indicator) present of the chip, maybe used for ASK demodulation or to ascertain the received signal strength. A maximum data rate of $115.2 \mathrm{kbps}$ can be achieved, with an output power of $4.5 \mathrm{dbm}$, quite suitable for moderately fast short distance communications. For our testing purposes we used two transceivers based on the reference design provided by Texas Instruments after minor variations. Both of them were enclosed in metallic enclosures and SMA (Sub Miniature A type) connectors provided connection to the antennae.

\section{CAUSES AND SOURCES OF ERRORS}

Wireless communication works on an essentially shared medium where a plethora of transmissions take place, which includes natural phenomena such as lightning. Although the spectrum has been divided into several bands, there is no certainty that a band may be unoccupied. For example, in case of frequency hopping systems, the same frequency may be in use by another device at a particular interval of time. As for binary data transfer, any unwanted flipping of a bit (binary digit) and / or a loss of a bit may be termed as an error.

\section{A. Design and orientation of Antenna}

A good antenna design has more effect on performance of a radio communication system than any other single part. The most critical parts of an antenna design are its placement and orientation. For the best performance between a transmitter and the receiver, the main lobes of each antenna must be aligned to point towards each other [6]. At $937.5 \mathrm{MHz}$ the wavelength (1) of an electromagnetic wave is $32 \mathrm{~cm}$, thus an $1 / 4$ antenna would have a length of $8 \mathrm{~cm}$, resulting in a compact design of the wireless modem.

\section{B. Distance and attenuation}

On an open and level ground, the effective received power at a distance is governed by the transmitted power and the receiver sensitivity. The maximum range of communication is determined by the relation given [1]:

$$
(\mathrm{Pt}-\mathrm{Pr})=37+20 \text { Log "f" (MHz)+20 Log "d" (miles) (1) }
$$

Where ' $\mathrm{Pt}$ ' is transmitted power in $\mathrm{dBm}$, ' $\mathrm{Pr}$ ' is the receiver sensitivity in $\mathrm{dBm}$, ' $\mathrm{f}$ ' is the carrier frequency in $\mathrm{MHz}$, and ' $\mathrm{d}$ ' is the maximum theoretical range in miles. TRF 6900A with an output power of $4.5 \mathrm{dbm}$ and $-85 \mathrm{dbm}$ receiver sensitivity, has a theoretical range of 2500 feet. It is difficult to get a direct line of sight within a room, due to such objects as furniture, partitions, etc which attenuate the radio waves, by virtue of absorption of energy leading to a drastic drop in the range. Human bodies also absorb radio waves and any movement in the vicinity of the device could lead to loss in received power, resulting in errors.

\section{Narrowband Interference}

A transmitter operating in the same frequency band as our device may cause interference and therefore errors [2]. In one of our tests we discovered interference, which was attributed to GSM handsets operating in the vicinity of the device after due scanning on the spectrum analyzer.

\section{Spread Spectrum Interference}

Devices operating in the ISM bands use spread spectrum methods primarily to avoid occupying a channel for long. Thus a channel collision can also lead to corrupted data, as the number of usable channels is limited by regulatory authorities [2], [8].

\section{E. Electromagnetic Interference}

Improper shielding of RF devices may lead to errors due to EMI (Electro Magnetic Interference), sources of which include utility power line, florescent lamps, microwave ovens, and various other electrical and electronic appliances. With the metallic cover removed off from our transceiver we 
detected sporadic errors when a fluorescent lamp fixed on the ceiling was switched on and off.

\section{E. Baseband encoding:}

Our device has a capability to handle both constant DC and NRZ encoded data, hence we tested its capability to precisely identify long sequence of 1's or 0's. Using NRZ encoding and subsequent training of the receiver, long series of zeros and ones were correctly detected. Without training any length above three consecutive zeros or ones were not recognized properly.

\section{F. Multipath fading}

This is caused by signals arriving at the receiving antenna in different phases. This effect is due to the fact that a signal may travel many different paths before arriving at the antenna. Some components of the original signal may travel to the receiver's antenna via a direct free space path. Others, which have been reflected, travel longer paths and reach the receiver with a delay, thereby creating an out-of-phase relationship between the two signals, affecting the received signal strength. Rayleigh fading [8] occurs when the difference in lengths of paths traveled is an odd multiple of half the wavelength of the carrier, leading to a complete loss of signal.

\section{G. Modulation at the transmitter}

The modulation index of an FSK modulator is defined by ' $h$ ', where

$$
\mathrm{h}=\mathrm{d} / \mathrm{Bm}
$$

' $d$ ' is the frequency deviation and ' $B_{m}$ ' is the bandwidth of the data to be transmitted. For a data rate of ' $R$ ' $k b p s$ the bandwidth ' $\mathrm{B}_{\mathrm{m}}$ ' is $\mathrm{R} / 2 \mathrm{KHz}$. The modulation index ' $\mathrm{h}$ ' should be within 0.5 and 1 to conserve bandwidth [7].

\section{THE TEST PROCEDURE}

A good test procedure intends to consider the important aspects related to errors, in all their permutations and combinations [2]. For the test process, we engaged a computer laboratory with thirty computers and lots of open space. The process involved setting up two transceivers, one at a fixed location, and moving the other one around the laboratory. One of the computers served as a transmitter and the other one a receiver. Following a test, the roles of transmitting and receiving were switched, with a due repetition of the same test. Various fine parameters such as phase noise, jitter, and DDS spurs were ignored, as we were more interested in macro effects. During the test only raw data was used to measure errors while error checking and retransmission schemes such as CRC (Cyclic Redundancy Check) or Checksum were disabled. We separated the modems by a distance of 5 meters for all distance independent tests.

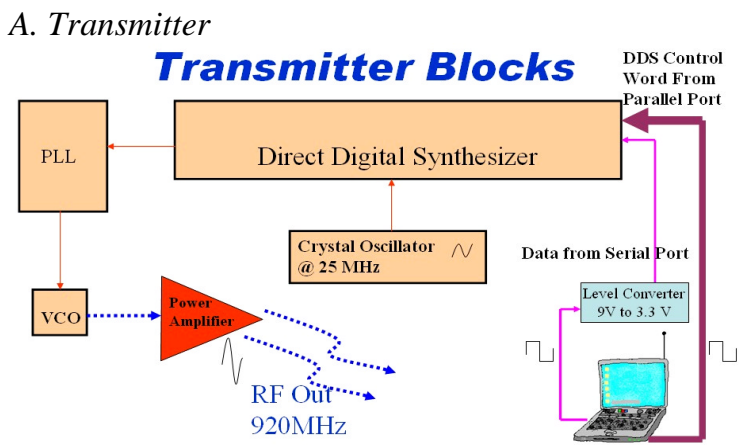

Fig. 1. Transmitter Setup

Our device is configured to transmit mode by sending the appropriate control word through the computer. Input loop filter bandwidth of the modem has been set to match the transmit data rate of $19.2 \mathrm{kbps}$. Wherever applicable in the tests, the default parameters include transmit frequency of $920 \mathrm{Mhz}$ with a frequency deviation of $30 \mathrm{KHz}$, and transmit power of $4.5 \mathrm{dBm}$. A quarter wave monopole antenna of length $8.15 \mathrm{~cm}$, with a measured return loss of $10 \mathrm{~dB}$, is connected to the device. The test data consists of a text file of size $30 \mathrm{~KB}$ with a transmit time of 15.6 seconds at $19.2 \mathrm{kbps}$. This size has been chosen so that we may get the file transmitted completely before the receiver 'store and hold' capacitor, used for NRZ data reception gets discharged, resulting in spurious errors. During the test runs the receiver performed quite well until about 30 seconds after which a training procedure was required, in order to charge the "store and hold' capacitor.

\section{B. Receiver}

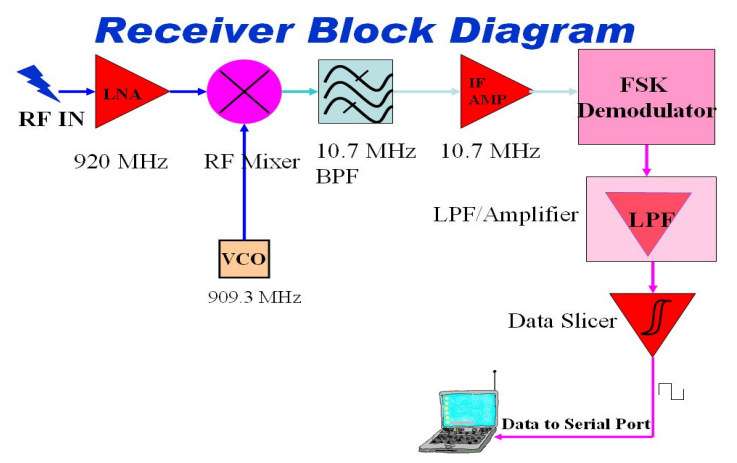

Fig. 2. Receiver Setup 
TRF6900A incorporates a super heterodyne receiver with an intermediate frequency (IF) of $10.7 \mathrm{MHz}$, due to which the receiver PLL is set to lock the VCO at $(920 \mathrm{MHz}-10.7$ $\mathrm{MHz}$ ) i.e. $909.3 \mathrm{MHz}$. A precise copy of the transmit antenna serves the receiver. The computer's serial port is configured at $19.2 \mathrm{kbps}$. The LNA has been set on high gain mode and the data slicer is first trained on an alternating sequence of zeros and ones, and on its completion is put on hold mode, to start receipt of NRZ encoded data. Data reception is temporarily stopped during the training period, and resumed thereafter. We measured the strength of the signal at the receiver using the RSSI which ranges from $0.44 \mathrm{~V}$ at $-80 \mathrm{dBm}$ input power to $2.6 \mathrm{~V}$ at $-10 \mathrm{dBm}$ input power, with a nominal slope of $19 \mathrm{mV} / \mathrm{dB}$.

\section{ENUMERATION OF THE TESTS}

\section{A. Path loss and attenuation test}

We keep the transmitter at a height of $2 \mathrm{~m}$ from the ground level, with the antenna oriented parallel to the floor. The receiver is moved to various distances away from the transmitter and the bit errors are measured at every location. Receiver antenna has the same orientation as that of the transmitter antenna. All other parameters are the default ones. The error rates are also measured with the transmit power attenuated by $10 \mathrm{~dB}$ and $20 \mathrm{~dB}$ respectively. Programming the chip can set these two attenuation levels.

\section{B. Baseband encoding test}

Our device supports both NRZ and Manchester encoded data, therefore it is useful to compare the effective error rates for both these schemes [3]. The catch in using NRZ encoded data is that the receiver needs to be trained after intervals of around half a minute, while Manchester encoded data may be received without any such procedures.
On the contrary NRZ encoding is twice as efficient as Manchester encoding, for the same bandwidth, resulting in faster data transfer. As an example, NRZ encoded data is transmitted at $19.2 \mathrm{kbps}$ while Manchester encoded data is transmitted at $9.6 \mathrm{kbps}$ both utilizing a bandwidth of $19.2 \mathrm{kbps}$

\section{Human body interference test}

Human bodies absorb radio waves, which may in turn produce errors, due to attenuation. Majority of the wireless transceivers end up in devices being used by people, thus the effect of a human body coming close to either the transmitter or the receiver should be important. In this test we measure the effect of people in vicinity of the receiver and the transmitter, on the error rates.

\section{E. Occlusion Test}

A metal and gypsum wall with a glass window served as our typical occlusion, as this is a popular partition present in many office scenarios. Our transmitter and receiver are placed on opposite sides of the wall, which runs from the floor to the ceiling.

\section{F. Modulation Test}

The recommended modulation index for a binary FSK transmitter ranges from 0.5 to 1. [4],[5],[7] Theoretically a deviation of $9.6 \mathrm{Khz}$ or more should suffice for a data rate of $19.2 \mathrm{kbps}$. TRF 6900A transceiver supports frequency deviation from $1.5 \mathrm{KHz}$ up to $398 \mathrm{KHz}$, thus supporting both narrowband and wideband FSK. We vary the deviation from $10 \mathrm{KHz}$ to $350 \mathrm{KHz}$, in ten steps and find out changes in error rates, while keeping distances and other parameters constant.

Table 1

Test Results for the TRF6900A device

\begin{tabular}{|c|c|c|c|l|l|}
\hline Test Name & $\begin{array}{c}\text { Bytes } \\
\text { Transferred }\end{array}$ & $\begin{array}{c}\text { Transmit } \\
\text { Power } \\
(\mathbf{d B m})\end{array}$ & $\begin{array}{c}\text { RSSI } \\
(\mathbf{V})\end{array}$ & $\begin{array}{c}\text { Errors } \\
(\text { Bits })\end{array}$ & \multicolumn{1}{|c|}{ Comments } \\
\hline Distance Test & & & & & The usable range of the device is \\
\hline Distance $=5 \mathrm{~m}$ & 150,000 & $-5.5 \mathrm{dBm}$ & 1.9 & 0 & well above 10 meters. \\
\hline Distance $=5 \mathrm{~m}$ & 150,000 & $-15.5 \mathrm{dBm}$ & 1.7 & 3 & \\
\hline Distance $=10 \mathrm{~m}$ & 150,000 & $4.5 \mathrm{dBm}$ & 1.8 & 0 & \\
\hline Distance $=10 \mathrm{~m}$ & 150,000 & $-15.5 \mathrm{dBm}$ & 1.5 & 4576 & \\
\hline
\end{tabular}




\begin{tabular}{|c|c|c|c|c|c|}
\hline $\begin{array}{l}\text { Baseband Encoding } \\
\text { Test }\end{array}$ & & & & & \multirow{3}{*}{$\begin{array}{l}\text { File consisted of concatenated series } \\
\text { of the byte ' } 00001111 \text { ' Error occurs } \\
\text { in Manchester mode mainly after the } \\
\text { third constant bit. }\end{array}$} \\
\hline NRZ Encoding & 150,000 & $4.5 \mathrm{dBm}$ & 1.9 & 0 & \\
\hline Manchester Encoding & 150,000 & $4.5 \mathrm{dBm}$ & 1.9 & 1391 & \\
\hline \multicolumn{5}{|l|}{ EMI Test } & \multirow[b]{2}{*}{$\begin{array}{l}\text { Substantial amount of errors due to } \\
\text { interference, even though the RSSI } \\
\text { level is nominal. }\end{array}$} \\
\hline $\begin{array}{l}\text { EMI generated due to } \\
\text { fluorescent lamps. }\end{array}$ & 150,000 & $4.5 \mathrm{dBm}$ & 1.9 & 1618 & \\
\hline \multicolumn{5}{|l|}{ Human Body Test } & \multirow{3}{*}{$\begin{array}{l}\text { Effect of human body is prominent } \\
\text { at the transmitter as well as the } \\
\text { receiver. }\end{array}$} \\
\hline Vicinity of receiver & 150,000 & $4.5 \mathrm{dBm}$ & 1.6 & 81 & \\
\hline $\begin{array}{l}\text { Vicinity of } \\
\text { transmitter }\end{array}$ & 150,000 & $4.5 \mathrm{dBm}$ & 1.6 & 107 & \\
\hline \multicolumn{5}{|l|}{$\begin{array}{l}\text { Modulation Test } \\
\text { (Frequency Deviation) }\end{array}$} & \multirow{6}{*}{$\begin{array}{l}\text { Too many errors with a modulation } \\
\text { index lower than } 3 \text {. } \\
\text { Obtained good performance with a } \\
\text { modulation index from } 3 \text { right up to } \\
31 \text {. } \\
\text { Modulation index in excess of } 31 \\
\text { again produces errors. }\end{array}$} \\
\hline Deviation $=10 \mathrm{KHz}$ & 150,000 & $4.5 \mathrm{dBm}$ & 1.9 & $\begin{array}{l}\text { Data } \\
\text { indiscernible }\end{array}$ & \\
\hline Deviation $=20 \mathrm{KHz}$ & 150,000 & $4.5 \mathrm{dBm}$ & 1.9 & 18,050 & \\
\hline $\begin{array}{l}\text { Deviation between } \\
30 \mathrm{KHz} \text { and } 250 \mathrm{KHz}\end{array}$ & 150,000 & $4.5 \mathrm{dBm}$ & 1.9 & 0 & \\
\hline Deviation $=300 \mathrm{KHz}$ & 150,000 & $4.5 \mathrm{dBm}$ & 1.9 & 64 & \\
\hline Deviation $=350 \mathrm{KHz}$ & 150,000 & $4.5 \mathrm{dBm}$ & 1.9 & 312 & \\
\hline \multicolumn{5}{|l|}{ Occlusion Test } & \multirow[b]{2}{*}{$\begin{array}{l}\text { Occlusion attenuates the signal } \\
\text { strength markedly, leading to errors. }\end{array}$} \\
\hline $\begin{array}{l}\text { Distance }=1 \mathrm{~m}, \text { with } \\
\text { occlusion in between. }\end{array}$ & 150,000 & $4.5 \mathrm{dBm}$ & 1.45 & 5128 & \\
\hline
\end{tabular}

\section{CONCLUSION}

A wireless device needs to be tested comprehensively before being put into application and our test method suffices to measure most of the macro parameters that may affect it. Few important facts may be inferred about the TRF 6900A as a result of the tests mentioned above.

a) Error is less than one per million bits at a distance of 10 meters in indoor situations with $4.5 \mathrm{dBm}$ output power.

b) Improper shielding of an RF device leads to errors due to EMI, which may be difficult to track down.

c) Human bodies near the vicinity of the modem generate errors. The effect is greater at the transmitter side than at the receiver.

d) Proper selection of the frequency deviation is important to conserve bandwidth and to minimize errors. Modulation index of ' 3 ' is the ideal choice for this device.

The software which has been used to test the modems would soon be put on the public domain, along with the detailed schematics and layout of our design.

\section{REFERENCES}

[1] Roy Blake, "Wireless Communication Technologies" Delmar Thomson learning, KY, US, 2001.

[2] D.Eckhardt, P.Steenkiste, "Measurement and Analysis of the Error Characteristics of an In-Building Wireless Network", Proceedings of ACM SIGCOMM '96, Stanford.

[3] Craig Bohren, Matthew Loy, and John Schillinger,

"TRF6900A Single-Chip RF Transceiver, Texas

Instruments Design Notes SWRA033D”, TX, US 2001.

[4] Loy Matt, "Understanding and Enhancing Sensitivity in Receivers for Wireless Applications, Texas Instruments application note, SWRA030”, TX, US, 1999.

[5] H.Taub and D.Schilling, "Principles of Communication Systems", NY, US, 1986.

[6] Tom Yestrebsky, "MICREL, MICRF001 Antenna Design Tutorial, Application Note 23", CA, US, 1999.

[7] Michael A. Soderstrand, Liz Gao and Earl McCune, "Maximizing Channel Capacity in FSK Modulation

Systems" Proceedings of ISCAS '99, Orlando, FL, US.

[8] “An Introduction to Wireless Technology, IBM, US,'95 